\title{
LA COMPRENSIÓN DE LOS POLÍGONOS POR MEDIO DEL GEOGEBRA EN ESTUDIANTES DE GRADO SÉPTIMO
}

\section{THE UNDERSTANDING OF POLYGONS THROUGH GEOGEBRA IN SEVENTH GRADE STUDENTS.}

\section{Deisy Tatiana Cuervo Lancheros ${ }^{1}$ \\ Carlos Andrés Fonseca Cuervo² \\ Omaida Sepúlveda Delgado³. \\ Universidad Pedagógica y Tecnológica de Colombia (Tunja, Colombia).}

\section{RESUMEN}

El presente artículo tiene como propósito presentar el desarrollo de un trabajo de investigación que tiene entre sus objetivos el reconocimiento de conceptos básicos de geometría por medio del diseño y aplicación de una pruebadiagnósticaque involucralautilización del software dinámico GeoGebra. La pregunta de investigación del estudio corresponde a: ¿Cómo las situaciones didácticas mediadas por el programa GeoGebra pueden conducir a los estudiantes de grado séptimo de una Institución Educativa a la construcción y comprensión de los polígonos regulares? Para dar respuesta a la informática para la docencia, Estudiante para las regiones - docentes y directivos docentes, de Instituciones Educativas Publicas de Boyacá, Convenio Especial de Cooperación No. 80740-687-2019 - Programa "BOYACÁ MAESTRA", Universidad Pedagógica y Tecnológica de Colombia. Tunja, Colombia. Email: carlosandres.fonseca@ uptc.edu.co Phone: +573123364232. ORCID ID: https://orcid.org/ 0000-0003-1168-7939

3 Docente Investigadora, Licenciada en Matemáticas, Ingeniera de Sistemas, Especialista en computación para la docencia, Magister en Ciencias Matemáticas y Doctora en Ciencias de la educación, Universidad Pedagógica y Tecnológica de Colombia, Tunja, Colombia. Email: omaida. sepulveda@uptc.edu.co Phone: +573118556222. ORCID ID: https://orcid.org/0000-0002-2950-8137 
pregunta planteada, inicialmente se aplicó una prueba a treinta estudiantes de grado séptimo de una Institución Educativa del departamento de Boyacá (Colombia). Se adopta en el estudio un enfoque cualitativo de tipo descriptivo, y se realiza el análisis de la información de acuerdo a las formulaciones de la Teoría de las Situaciones Didácticas y el modelo de Van Hiele. Entre los resultados evidenciados a partir de la implementación de esta prueba diagnóstica, se identificaron algunas dificultades especialmente, en el desarrollo en forma autónoma y completa de situaciones problema y el trabajo en grupo, relacionado con el intercambio y la comparación de la información con los compañeros. Por tanto, luego de los análisis a las situaciones planteadas, se concluye que es importante implementar en el diseño de estas secuencias didácticas, situaciones adidácticas, ya que estas permiten la construcción autónoma del saber matemático, y fortalecen el aprendizaje geométrico y el trabajo en grupo.

\section{PALABRAS CLAVE:}

GeoGebra, segmentos, polígono irregular, polígono regular, distancia.

\section{ABSTRACT}

The objective of this research article is the recognition of basic geometry concepts through the design and application of a diagnostic test that involves the use of the dynamic software GeoGebra. This test is part of a research study in which the research question is posed: ¿How can didactic situations mediated by the GeoGebra program lead seventh grade students in an educational institution to the construction and understanding of regular polygons? The test was applied to thirty seventh grade students of an Educational Institution in the department of Boyacá (Colombia).

A descriptive qualitative approach is adopted in the study, and the analysis of the information is carried out according to the assumptions of the Theory of Didactic Situations and Van Hiele's model. Among the results evidenced from the implementation of the diagnostic test are some of the students' difficulties in the autonomous and complete development of the proposed problem situation and others related to the exchange and comparison of information with other students.

KEYWORDS: GeoGebra, segmets, irregular polygon, regular polygon, distance

\section{INTRODUCCIÓN}

La tecnología ha sido hasta el momento utilizada en diferentes sectores con finalidades productivas, su incorporación se ha dado de manera rápida evidenciando resultados favorables, por esto las Tecnologías de la Información y las comunicaciones (TIC) aportan a los países mejores condiciones laborales y fortalecen constructivamente la competitividad entre las industrias y diferentes sectores públicos (MEN, 2006).

En particular, en el sistema educativo, se evidencia que este no puede seguir utilizando los métodos de enseñanza que desde años anteriores se han venido estructurando debido a la situación originada por la pandemia; así, la educación del hoy debe buscar nuevas alternativas que se encuentren en función de las TIC generando desafíos y expectativas para el logro completo y la transformación de los procesos educativos (Said, 2015).

EI MEN (2006) hace énfasis en especificar la estrecha relación entre el sistema educativo y las TIC en función de obtener excelentes resultados en la educación de los estudiantes: "Las TIC no sólo ponen al alcance de docentes y estudiantes volúmenes de información, sino que promueven el desarrollo de destrezas y habilidades como son la búsqueda, selección y procesamiento de información, así como la capacidad para el aprendizaje autónomo" (p.49).

En este sentido, Córdoba (2014) en su trabajo sobre las TIC en el aprendizaje de las matemáticas, plantea que: "con la llegada de 
estas tecnologías se han sentado diversas posturas en cuanto a su incorporación e integración curricular $\mathrm{y}$ a las ventajas o desventajas que podrían tener en el desempeño académico de los estudiantes" (p.2).

Enfatizando en el área de matemáticas se menciona que alrededor de esta existen varias creencias de los estudiantes en cuanto al proceso de enseñanza y aprendizaje de la misma, como el hecho de que es la asignatura más difícil y la materia que todos los estudiantes pierden, llevando incluso en algunos casos a que los estudiantes seleccionen una formación profesional ajena a la matemática (Leung, 2006). Según los argumentos, es necesario incorporar nuevos mecanismos y herramientas que incentiven y generen mejores resultados en los estudiantes, como el caso de las TIC, que han presentados grandes avances e innovaciones en estos últimos años para la matemática.

Una de las ramas de la matemática en la que ha irrumpido con gran fuerza el uso de las TIC ha sido la geometría, esta se caracteriza fundamentalmente porque permite percibir las formas del espacio en donde se vive y describir sus características. Vargas \& Gamboa (2013) acerca de la geometría ofrecen una percepción: "La geometría es para el ser humano el idioma universal que le permite describir y construir su mundo, así como transmitir la percepción que tiene de este al resto de la humanidad" (p.75).

En este aspecto, se puede pensar que una estrategia a considerar para mejorar el proceso de enseñanza-aprendizaje de la geometría es la incorporación del uso de Software de Geometría dinámica en las clases como el Geoemter's Skechpad, Geup, Cabri II y el GeoGebra, ya que estos programas ofrecen diferentes herramientas que permiten dinamizar cualquier objeto matemático y permiten hacer geometría de una manera activa.
En especial el GeoGebra, brinda diferentes herramientas para la representación de cualquier objeto matemático y permite que el estudiante consolide los conceptos y se promueva la participación de los mismos en la construcción de figuras planas que observa en su entorno. Además, permite crear ambientes virtuales de aprendizaje en donde el estudiante se vuelve protagonista de su aprendizaje, y aprende a ver los conceptos matemáticos de manera tangible, con la posibilidad de explorarlos y manipularlos. Así mismo al docente le permite identificar la manera como razona el estudiante, cómo utiliza lo que sabe para la resolución de problemas y cómo utiliza las herramientas para plantear soluciones (Ezguerro, 2014).

Los anteriores argumentos están expuestos en el trabajo de investigación que se encuentra en desarrollo "El GeoGebra para la comprensión de los polígonos regulares en estudiantes de grado séptimo". El objeto matemático de estudio para este artículo de investigación son los polígonos y en particular el reconocimiento de conceptos básicos por medio del software dinámico GeoGebra. En este documento se presenta el análisis a la implementación de la actividad diagnóstica denominada: "Reconocimiento de conceptos básicos y polígonos" esto, como un primer acercamiento de los estudiantes al desarrollo de actividades fundamentadas en la Teoría de las Situaciones Didácticas y el modelo de Van Hiele. Para esta prueba diagnóstica, se trabajó con estudiantes de grado Séptimo de una Institución Educativa del departamento de Boyacá (Colombia). Para el análisis de la prueba se presenta el marco teórico, la metodología y se describen los resultados encontrados con la discusión respectiva, para llegar a las conclusiones relacionadas con los objetivos de la prueba diagnóstica.

\section{MARCO TEÓRICO}

Esta investigación se enmarca en un enfoque constructivista del proceso de enseñanza y 
aprendizaje de las matemáticas en el aula de clase; se centra en la visión de promover investigación matemática en los estudiantes de acuerdo a las habilidades de los mismos. Por tanto, se considera que las teorías de la didáctica de la matemática que respaldan la investigación son: la Teoría de las Situaciones Didácticas y el modelo de Van Hiele.

\section{TEORÍA DE LAS SITUACIONES DIDÁCTICAS}

La Escuela Francesa de la didáctica de la matemática desde sus inicios en los años setenta (70), se ha preocupado por la necesidad de descubrir fenómenos relacionados con la adquisición y trasmisión de conocimientos matemáticos por parte de los estudiantes dentro y fuera de las aulas de clase, además ha contribuido de manera significativa al aporte del desarrollo de teorías en la enseñanza de las matemáticas con la estructuración de diseños constructivistas. Entre sus representantes se encuentra Guy Brousseau el cual es su creador.

Brousseau (2007) indica que "la enseñanza es concebida como las relaciones entre el sistema educativo y el alumno vinculadas a la transmisión de un saber dado y, de este modo, la relación didáctica se interpreta como una comunicación de informaciones" (p. 13). Esta concepción está relacionada con la idea que el maestro organiza el saber a enseñar al alumno y esté toma lo necesario.

Dentro de la estructura de la Teoría de situaciones didácticas, Brousseau (2007) define conceptos fundamentales para ser aplicados por parte de los docentes de matemáticas.

\section{SITUACIÓN DIDÁCTICA}

La cual describe la actividad del profesor, del alumno y del mismo sistema educativo. El aprendizaje que se adquiere se logra por medio de una adaptación del sujeto que aprende al medio, creado en la situación planteada, con intervención o no del docente en el transcurso del proceso (Brousseau, 2007, p.18). El profesor proporciona el medio didáctico con el fin que el estudiante construya su conocimiento, manifestando la relación de los elementos del triángulo didáctico.

\section{SITUACIÓN ADIDÁCTICA}

Una situación adidáctica se considerada como el aspecto donde la intención de enseñanza no aparece explicita para el alumno. Sólo interviene el estudiante y el medio. El docente propone problemas, de manera que el alumno pueda aceptarlos por medio de una reflexión y análisis de los mismos. El docente no interviene hasta que el estudiante produce la respuesta. Así, se denomina situación adidáctica como lo argumenta Brousseau al momento en el que: "El estudiante no habrá adquirido verdaderamente el conocimiento hasta no ser capaz de utilizarlo en situaciones que encuentre fuera de todo contexto de enseñanza y en ausencia de cualquier indicación intencional" (Brousseau, 2007, p. 31)

\section{CONTRATO DIDÁCTICO}

Se "refiere a la consigna establecida entre profesor y alumno, de esta forma, comprende el conjunto de comportamientos que el profesor espera del alumno y el conjunto de comportamientos que el alumno espera del docente" (Chavarría, 2006, p.3).

\section{VARIABLES DIDÁCTICAS}

Como lo expone Brousseau (2007): las variables didácticas son determinadas por el profesor, además son elementos del problema planteado al estudiante por el profesor, es decir, modificadas por el docente de tal forma que se requiera un cambio en cada una de las estrategias de resolución de la situación planteada, lo cual permitirá a los estudiantes resolver diferentes problemas que utilicen un mismo conocimiento. 


\section{TIPOLOGÍA DE LAS SITUACIONES DIDÁCTICAS}

Situación de acción: en esta situación interactúa e intervienen el sujeto (estudiantes) y el medio. Además, el alumno actúa frente al problema propuesto utilizando sus conocimientos previos, descubriendo, experimentando, aceptando y rechazando diferentes estrategias de solución de la problemática diseñada y planteada por el docente (Brousseau,2007). Se afirma que una buena situación de acción debe permitir al alumno juzgar los resultados sin la intervención del profesor Chevallard, Bosch, \& Gascón (2005). De manera similar Brousseau (2007) indica que: "la sucesión de situaciones de acción constituye el proceso por el cual el alumno va a "aprenderse" un método de resolución de su problema" (p.22).

Situación de formulación: Esta situación, corresponde a la capacidad del sujeto para identificar, descomponer y reconstruir en un sistema lingüístico el problema, además el mismo medio exige en la formulación involucrar a otro sujeto, con el fin de intercambiar la información encontrada en un lenguaje matemático de acuerdo al objeto matemático establecido (Brousseau, 2007).

Situación de validación: situación de discusión, donde el estudiante debe probar que el modelo elegido es válido. Brousseau (2007) indica que: "El alumno no sólo tiene que comunicar una información, sino que también tiene que afirmar que lo que dice es verdadero en un sistema determinado, sostener su opinión o presentar una demostración" (p. 23).

Situación institucionalización: como lo sustenta Brousseau (2007) los alumnos formalizan el conocimiento matemático, se obtienen conclusiones y se sistematiza lo aprendido en el desarrollo de las secuencias didácticas en la construcción de saberes.

\section{EL MODELO DE VAN HIELE}

En este modelo se establecen cinco niveles para la comprensión de los objetos matemáticos, donde superando un nivel se puede pasar al siguiente. Estos niveles son cuatro (Fouz, 2013).

Nivel 0. Visualización y reconocimiento (familiarización): los objetos se perciben en su totalidad como un todo, no hay diferencias de características y componentes, los objetos se describen y no se reconocen las propiedades del objeto de estudio.

Nivel 1. Análisis (comparación): en este nivel se reconocen las propiedades del objeto de estudio a partir de la observación y experimentación, de manera informal se describen las figuras por sus propiedades, pero no se relacionan ni figuras ni propiedades y no se elaboran definiciones.

Nivel 2. Ordenación o clasificación: en este nivel se describen las figuras de manera formal, lo cual implica entender el significado de las definiciones, se realizan clasificaciones lógicas de manera formal, ya que el nivel de razonamiento matemático ya está iniciado.

Nivel 3. Deducción formal (argumentación): en este punto se realizan demostraciones lógicas formales, permitiendo justificar adecuadamente las proposiciones. De igual forma, en este nivel se comprende la naturaleza axiomática de las matemáticas y se manejan las relaciones entre las propiedades.

Nivel 4. Rigor: se reconoce que existen diversos sistemas axiomáticos, los cuales se pueden comparar, permitiendo realizar una geometría de manera abstracta, es decir, sin necesidad de ejemplos concretos, y así alcanzar un alto rigor matemático.

La evaluación en el modelo de Van Hiele es un factor importante en la secuenciación de cada uno de los niveles, se tiene en cuenta el nivel de razonamiento de los alumnos, se valora la forma 
cómo los alumnos responden y el porqué de cada una de las respuestas más que lo que no contestan o responden bien o mal (Fouz, 2013).

\section{METODOLOGÍA}

La presente investigación tiene un enfoque cualitativo, el cual permite recolectar datos con el fin de fundamentar el proceso con la interpretación y reflexión de los mismos. Desde este punto de vista y de acuerdo con Hernández, Collado \& Baptista (2014) la investigación cualitativa parte de lo especifico a lo general, es decir, es un proceso inductivo, que permite explorar y describir; se fundamenta principalmente en la interpretación y el análisis de los datos.

Es se tipo descriptiva como lo sustenta Arias (2012):

La investigación descriptiva consiste en la caracterización de un hecho, fenómeno, individuo o grupo, con el fin de establecer su estructura o comportamiento. Los resultados de este tipo de investigación se ubican en un nivel intermedio en cuanto a la profundidad de los conocimientos se refiere. (p. 24).

Se hace énfasis en el uso de variables didácticas que fomentan el aprendizaje del objeto de estudio. Como variables micro didácticas se tiene el uso de las herramientas del software GeoGebra como la herramienta punto, la herramienta segmento, la herramienta polígono regular, la herramienta distancia y la herramienta mover o seleccionar un objeto, el uso de estas herramientas permitió el reconocimiento de algunos conceptos básicos de geometría en especial el de polígono.

La población objeto de estudio estuvo conformada por todos los estudiantes de grado séptimo matriculados en el año escolar 2021 en la Institución Educativa. El grado séptimo A, conformado por 15 mujeres y 15 hombres y el grado séptimo B conformado por 12 mujeres y 19 hombres. La Institución Educativa es de carácter público y se encuentra ubicada en el departamento de Boyacá. Este colegio ofrece educación en preescolar, básica primaria, básica secundaria y media a estudiantes del municipio.

En relación con la muestra, participaron 30 de los 61 estudiantes, 13 estudiantes de séptimo A y 17 estudiantes de séptimo B, participando voluntariamente en el trabajo con el desarrollo de la prueba diagnóstica objeto de este estudio.

Debido a la situación actual generada por la pandemia del Covid 19 en Colombia, la educación ha sufrido diversos cambios a los cuales las Instituciones Educativas han tenido que adaptarse y de la misma forma desarrollar estrategias para continuar con el proceso educativo de los estudiantes. En la Institución Educativa mencionada, se trabaja con la estrategia: "A estudiar en casa" para esto se utilizan guías de aprendizaje, que se diseñan según lo establecido en el plan de área de matemáticas, para garantizar que todos los estudiantes tengan acceso y continuidad en el proceso de aprendizaje de las matemáticas.

El acompañamiento en la asignatura de matemáticas en grado séptimo se realiza por medio de la aplicación WhatsApp y llamadas telefónicas: al iniciar la semana se dan las indicaciones sobre el trabajo a realizar en la guía establecida, se comparte videos tutoriales sobre el tema que se aborda en la guía y para tutorías o para resolver dudas se utilizan los horarios de clase establecidos para matemáticas. Este proceso de enseñanza y aprendizaje se ve afectado por aspectos externos como la falta de motivación de algunos estudiantes, la falta de acceso a datos o internet por parte de algunos estudiantes y el acceso a un celular $o$ un computador.

En cuanto a los instrumentos de recolección de información se utiliza la prueba diagnóstica: 
"Reconocimiento de conceptos básicos y polígonos" la cual busca identificar las concepciones y conocimientos previos de los estudiantes de grado séptimo sobre el objeto polígonos.

\section{RESULTADOS}

En el marco del desarrollo de esta actividad diagnóstica, primero se realiza una sesión introductoria al manejo del software dinámico GeoGebra, para esto se solicita con anticipación a los estudiantes que descarguen la aplicación GeoGebra Geometría, en el celular, ya que la mayoría de la población cuenta con celular y no con computador.

Después se programa una clase por WhatsApp, para explicar el uso de GeoGebra, a partir de la elaboración de videos tutoriales sobre el manejo y funcionalidad de las herramientas básicas del programa, tales como mover o seleccionar un objeto, punto, segmento, rectas, circunferencias, distancia o longitud y la herramienta polígono regular. Después de observar los videos tutoriales se pide a los estudiantes desarrollar algunas actividades sencillas para que interactúen y manipulen las herramientas explicadas.

Para el desarrollo de la actividad diagnóstica se trabajó de forma asincrónica debido a las condiciones expuestas sobre las dificultades de conectividad de los estudiantes: se envía la guía de aprendizaje y se dispone de tres días para que los estudiantes desarrollen y presenten las evidencias del desarrollo de la actividad. A continuación, se presentan los resultados según la propuesta de la Teoría de las Situaciones didácticas y el modelo de Van Hiele:

\section{SITUACIÓN DE ACCIÓN}

En esta situación se analiza si el estudiante trabajó de manera activa y si mostró buena disposición para realizar la situación problema propuesta; si hace uso de los conocimientos previos y si sigue las indicaciones dadas en la guía e interactúa con las herramientas del programa.

Por tanto, se evidencia que la mayoría de los estudiantes marcan los cinco puntos en la ventana de trabajo del GeoGebra y los unen por medio de segmentos construyendo pentágonos irregulares. Los estudiantes relacionan esta construcción del pentágono irregular como la forma de una casa, como lo establece E13 en su construcción.

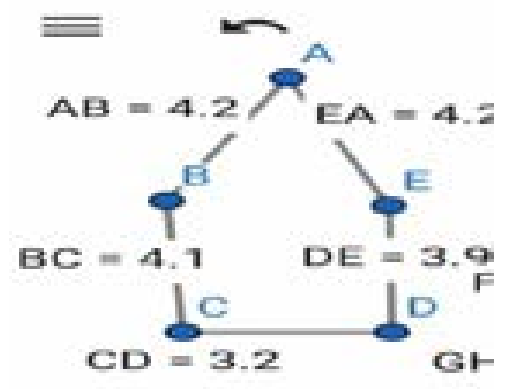

Figura 1. Situación acción E13

La estudiante E3 marca los cinco puntos, pero al unirlos por medio de segmentos, solo une cuatro puntos y no cierra la figura, por lo que construye una figura plana abierta, E19 construye también una figura abierta. A continuación, se observa la construcción de E3.

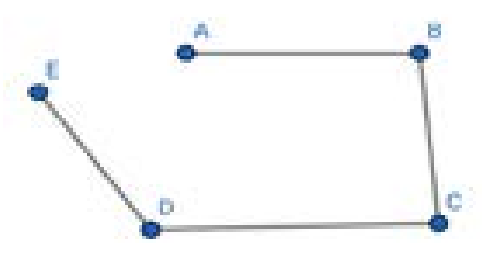

Figura 2. Situación acción E3

El estudiante E8 marca siete puntos y construye un heptágono irregular, E12 marca cuatro puntos y los une con segmentos, construyendo un rectángulo, E16 y E23 marca seis puntos, el primero une los puntos por segmentos y construye un hexágono irregular, mientras que el segundo une los seis puntos trazando 
el contorno de una estrella, como se ilustra a continuación:

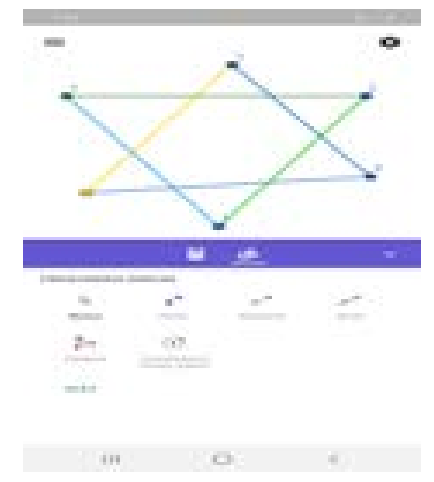

Figura 3. Situación acción 23

El estudiante E11 no construye ninguna figura por medio de segmentos.

En cuanto a la construcción de un polígono utilizando la herramienta polígono regular, la mayoría de los estudiantes construyeron hexágonos regulares, luego en su orden cuadrados, octágonos, triángulos y pentágonos: E6 fue el único estudiante que construyó un pentágono regular y E21 un decágono. E9 no construye ningún polígono regular.

De los anteriores resultados se concluye que la mayoría de los estudiantes que participaron en esta situación, cooperaron de manera activa y siguieron las indicaciones establecidas, solo unos pocos, realizaron construcciones ajenas a lo establecido. En este proceso, se evidenció que algunos estudiantes solicitaban explicación al docente sobre qué hacer, cómo realizarlo y qué herramientas utilizar, es decir, no deseaban trabajar por su propia iniciativa. Respecto a los saberes previos sobre el tema, se concluye que la mayoría de estudiantes los utilizaron y únicamente en dos estudiantes se evidenció que no se había comprendido la situación propuesta debido a que no se realizaron las construcciones indicadas.

Respecto al Nivel de familiarización, propuesto en el modelo Van Hiele, se establece que los estudiantes manipularon las herramientas del programa GeoGebra como punto, segmento y polígono regular. Algunos estudiantes (5) marcaron más o menos de cinco puntos, la mayoría de estudiantes construyeron figuras planas cerradas por medio de segmentos, y en su totalidad trataron de seguir las indicaciones establecidas según este nivel.

\section{SITUACIÓN DE FORMULACIÓN}

En esta situación se tiene en cuenta la forma en cómo el estudiante intercambia información, argumenta, compara y comunica con otro estudiante los resultados encontrados. Por esto, se propuso conformar grupos de dos estudiantes, debido a la facilidad de ellos para que se comunicaran e interactuarán vía WhatsApp para realizar las respectivas comparaciones. El trabajo grupal en esta situación fue asincrónico debido a que no había una comunicación directa entre los estudiantes, los grupos se conformaron de la siguiente manera: Grupo 1 (E1-E2), grupo 2 (E3-E4), grupo 3 (E5-E6), grupo 4 (E7-E8), grupo 5 (E9-E11), grupo 6 (E10-E12), grupo 7 (E13-E14), grupo 8 (E15-E16), grupo 9 (E17-E18), grupo 10 (E19-E22), grupo 11(E20-E21), grupo 12(E23-E25), grupo 13 (E24-E26), grupo 14(E27-E29) y grupo 15 (E28-E30).

En el grupo 1, los estudiantes midieron las longitudes de los lados de la figura construida con segmentos y el polígono regular; realizaron las comparaciones de cada medida y registraron la información, socializando cada medida. El estudiante E1, para la escritura de los segmentos comprendidos entre dos puntos utilizó una letra mayúscula y otra minúscula y E2 omitió la escritura del signo igual, pero ninguno de los dos identificó los errores cometidos.

En el grupo 2, 3, 7, 9, 11 y 13 los dos estudiantes realizaron la comparación de sus construcciones y de los datos de la longitud de cada lado de la figura construida con segmentos y la construida con la herramienta polígono regular, registraron 
la información obtenida de la medida de cada lado en las tablas proporcionadas.

En el grupo 4, E7 midió la longitud de los lados del pentágono irregular construido, pero no registró la información en la tabla y tampoco registró los datos del compañero, en la construcción del triángulo equilátero mide la longitud de cada lado, pero registra datos que no corresponden, lo mismo sucede con los datos del compañero. Por su parte, E8 mide la longitud de los lados del heptágono irregular, compara la información, la registra en la tabla, pero, en la escritura de la información omite escribir el signo igual y escribe Para los datos del polígono regular en la escritura de la información E8 omite escribir el signo igual y omite registrar la medida de tres lados, lo escribe de la forma: Ninguno de los dos estudiantes determina los posible errores cometidos en la escritura de la información matemática.

En el grupo 5, E9 mide cada lado del polígono construido con segmentos, no compara con ningún compañero y únicamente registra en la tabla lo datos, mientras que para el polígono regular no mide la longitud de cada lado, únicamente se limita a copiar la información del compañero. E11 solo mide la longitud de cada lado del octágono, no compara con el compañero, y registra la información que observa de su construcción.

En el grupo 6, E10 de manera correcta mide la longitud de cada lado de la figura con segmentos y el polígono regular, luego compara con el compañero las construcciones y medidas y registra la información. E12 mide la longitud de cada lado, compara la construcción y los datos con el compañero y registra la información de manera incorrecta: y para el polígono regular registra como valores de los segmentos de los lados: .
En el grupo 8, se identifica que no se trabajó colaborativamente, porque E15 no comparó las construcciones ni medidas con E16, y este último se limitó a medir la longitud de cada lado de las figuras construidas y no comparó. Situación similar se presentó en el grupo 12 y el 15, donde los estudiantes E23, E25, E28 y E30 no trabajaron en grupo de acuerdo a las indicaciones dadas y registraron solo las longitudes de cada lado de las construcciones que realizaron.

Por su parte en el grupo 10, el estudiante E19 mide la longitud cada lado de la figura abierta que construye y compara los datos con su compañero E22, ninguno de los dos identifica que no es una figura plana cerrada y proceden a comparar las medidas de las figuras construidas.

En particular en el grupo 14, E29 no desarrolló lo propuesto en la situación y E27 diligenció las medidas de cada lado de la figura que construyó con segmentos, pero omite escribir la notación de segmento como lo proporciona el GeoGebra y registra la información de forma incorrecta como y no mide la longitud de cada lado del cuadrado que construyó.

En el Nivel de comparación, la mayoría de los estudiantes diferenciaron cada objeto construido, como la figura formada por segmentos y la figura construida con la herramienta polígono regular, y menos de la mitad de los estudiantes (8) identificaron que cada lado de la figura construida con segmentos tiene una longitud diferente, esto al utilizar la herramienta distancia.

\section{SITUACIÓN DE VALIDACIÓN}

Para esta situación se tiene en cuenta la forma cómo el estudiante argumenta y defiende correctamente el conocimiento adquirido frente a los demás compañeros. Para esto, se programó una sesión de clase por WhatsApp para los 30 estudiantes participantes de la actividad, en cada uno de los grupos se escogió un representante 
para socializar los resultados obtenidos con respecto a la longitud de los lados del polígono construido con segmentos y los resultados de la longitud de cada lado del polígono regular.

Los representantes de los grupos 1, 2, 4, 6, 7, 9 y 11 coincidieron en argumentar que la longitud de los lados del polígono construido con segmentos tiene longitud diferente a cada lado del polígono regular, porque la longitud de los lados del primer polígono varía en cada uno de sus lados por ser un polígono irregular, y en cambio las medidas de los lados del segundo polígono eran exactamente iguales por tratarse de un polígono regular.

Al respecto la estudiante E14 señala que: "El polígono construido por segmentos tiene su estructura de una manera desorganizada, por otro lado, el polígono construido con la herramienta polígono regular tiene su estructura más organizada" esto, refiriéndose a la forma de las construcciones. E4 agrega que: "Las medidas de la figura construida con segmentos no son iguales porque son polígonos irregulares y cada lado mide diferente". En cambio, E20 expresa que: "en el polígono regular todas sus longitudes son iguales y de la misma medida"

Por otra parte, E6 en representación del grupo 3 establece que "son de diferente longitud" sin especificar a qué construcción se refiere. De manera similar E25 argumenta que: "La diferencia es que las longitudes, los ángulos son diferentes." De esta manera E22 indica que: "son diferentes figuras, porque cada una es construida con una herramienta diferente". El vocero del grupo 13 menciona que: "Los resultados fueron bastantes diferentes, ya que el polígono construido con segmentos no es igual a los lados del polígono regular". Por su parte, E27 del grupo 14 seña: "con segmentos es un poquito más difícil y con polígonos es más fácil" y E30 del grupo 15 agregó que "los resultados fueron favorables".
Los representantes de los grupo 5 y 8, no participaron en la socialización. De esta manera se logró identificar que en esta situación de validación más de la mitad de los estudiantes (22) cumplieron con el objetivo de la actividad propuesta fomentando un trabajo interactivo entre ellos mismos.

En el Nivel de clasificación, se buscaba identificar cómo el estudiante realiza clasificaciones de los objetos geométricos manipulados y construidos y los relaciona con objetos de su entorno.

Los estudiantes buscaron objetos de su entorno donde se encontraban figuras similares a las construidas y trataron de realizar una representación de las mismas, como en el caso del polígono regular, donde los estudiantes lo asocian con una casa sencilla, un cuadro enmarcado, una ventana, una mesa rectangular, un reloj de pared que tiene forma octagonal, un cuadro de pintura, un televisor, las baldosas hexagonales del piso, una cometa en forma de cuadrado y triángulo, un kiosco, una lámpara, la pared de la casa, un enchape en forma pentagonal y un balón de forma hexagonal y no cuadrado. En este nivel se destaca que el estudiante E18, utilizó hojas de una planta para construir un octágono y un heptágono irregular, como se observa en las figuras $4 \mathrm{y}$ 5 evidenciando creatividad y la utilización de elementos de su entorno para desarrollar la actividad propuesta.

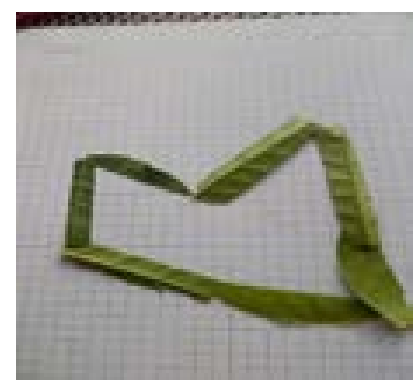

Figura 4. Heptágono irregular 


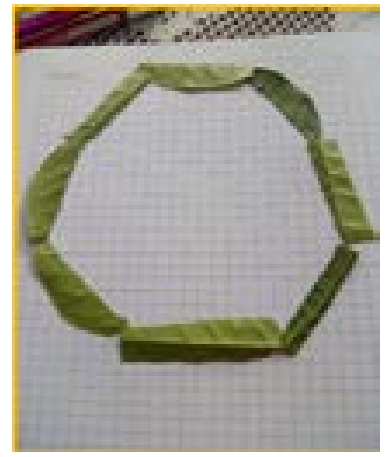

Figura 5. Octágono irregular

\section{SITUACIÓN DE INSTITUCIONALIZACIÓN}

Después de escuchar y leer las argumentaciones de los estudiantes, la docente compartió un video donde se presentaban las definiciones de polígono irregular y de polígono regular como complemento a lo expresado por los estudiantes, de esta manera se formalizó el conocimiento.

En el Nivel de argumentación, los estudiantes presentaron algunas conclusiones basadas en las propiedades observadas en el desarrollo de la situación didáctica, entre las más relevantes se encuentra la de E1: "si se mueve uno de los vértices con la herramienta seleccionar objeto, el polígono cambia de longitud, pero cada lado conserva la misma longitud del otro, lo que cambia es la longitud total de la figura"; de igual forma, E14 justificó: "al mover el vértice del polígono las longitudes de los lados cambian; si lo movemos para afuera mide más, pero si lo movemos para adentro mide menos". E17 mencionó que: "cuando uno disminuye el tamaño del polígono disminuye la longitud de cada lado y si se aumenta el tamaño del polígono aumentan las longitudes de los lados" y E20 argumentó: "Al mover un vértice podemos aumentar y disminuir la longitud de los lados del polígono".

\section{DISCUSIÓN}

En la situación de acción se identificó que la mayoría de los estudiantes desarrollaron la situación propuesta, interactuaron con el medio y tuvieron una buena disposición, además se evidenció que la mayoría de los estudiantes utilizan los saberes previos y realizan una devolución de la situación propuesta, de esta manera, se verifica lo expresado por Brousseau (2007) según el cual el alumno debe actuar frente al problema propuesto utilizando sus conocimientos previos, descubriendo y experimentando. De manera similar, Gómez, Sánchez, \& Sepúlveda (2021) mencionan en su trabajo de investigación que el diseño de diferentes situaciones problema, fomenta en los estudiantes el aprendizaje de conceptos a partir de los saberes previos, favoreciendo de esta manera la construcción de aprendizajes significativos.

Según Chevallard et al. (2005) una buena situación de acción, debe permitir al alumno juzgar los resultados sin la intervención del profesor. Respecto a lo anterior, en la prueba se identificó la falta de autonomía de menos de la mitad de los estudiantes ya que siempre estuvieron preguntando lo que debían hacer y cómo hacerlo, evidenciando falencias en las competencias interpretativa y propositiva de lo presentado en la situación de acción y buscando realizar un trabajo dependiente de las respuestas de la docente, resultado que se puede considerar emergente del contrato didáctico que siempre se ha establecido en la mayoría de las clases de matemáticas. Es por esto, que en las aulas de clase de matemáticas se debe fomentar la construcción de aprendizajes significativos en los estudiantes por medio del diseño y aplicación de situaciones adidácticas.

Un factor relevante manifestado por los estudiantes en la situación de formulación fue la falta de trabajo cooperativo en los grupos establecidos y las constantes deficiencias en la comunicación, esto debido en su mayoría a la falta de interacción entre los estudiantes 
de manera presencial en el aula de clase y la imposibilidad de realizar un trabajo virtual.

De igual forma, menos de la mitad de los estudiantesidentificaron sus erroresy dificultades en la escritura de las medidas de cada lado de acuerdo a la información proporcionada por el programa, ya que no trabajaron de acuerdo a las indicaciones establecidas, y algunos estudiantes en esta situación aún no lograron tener un trabajo autónomo, ya que siempre, preguntaban al docente lo que se debía realizar, además un estudiante no realizó lo propuesto en esta situación, de esta forma, no se evidencia en gran totalidad lo propuesto por Brousseau (2007) sobre la situación de formulación, según la cual, los estudiantes deben reconstruir en un sistema lingüístico la situación propuesta e intercambiar la información hallada por medio de un lenguaje matemático con otros estudiantes.

Por otra parte, según lo expuesto por los estudiantes en la situación de validación, el desarrollo de lo propuesto en las anteriores situaciones, permitió la socialización de resultados favorables; lo anterior debido a que la mayoría de los representantes de cada grupo manifestaron identificar que las longitudes de cada lado del polígono construido con segmentos son diferentes en comparación a las medidas iguales de cada lago del polígono regular, de esta manera, y de acuerdo a Brousseau (2007) la mayoría de los grupos, excepto dos, argumentaron los resultados encontrados en base a las construcciones realizadas.

De igual forma, en la situación de institucionalización, Brousseau (2007) menciona que los alumnos formalizan el conocimiento matemático, se obtienen conclusiones y se sistematiza lo aprendido. Al respecto, los estudiantes manifestaron haber comprendido en totalidad la definición de polígono irregular y la definición de polígono regular, confirmando así, los resultados expuestos por la gran mayoría, y complementando con conclusiones que establecieron de acuerdo al desarrollo de la situación propuesta.

En particular, en relación con los niveles propuestos por el modelo Van Hiele como lo expone Fouz (2013), los estudiantes realizaron una construcción de los objetos geométricos indicados, además reconocieron algunas propiedades de las figuras construidas a partir de la experimentación y observación, y se evidencia que se establecen clasificaciones no lógicas ni formales en relación a las construcciones realizadas y se plantean algunas argumentaciones.

Finalmente, respecto a la Teoría de las Situaciones Didácticas y en especial al diseño de situaciones adidácticas, estas permitieron a los estudiantes movilizarse por cada situación con el fin de determinar una construcción propia del conocimiento sobre el objeto matemático a estudiar: al respecto Fandiño (2009) sugiere: "usar, en lo posible, situaciones adidácticas al menos en los puntos más significativos. Las situaciones adidácticas dan garantía de construcción significativa de competencia, las didácticas, pueden incluso impedir la construcción de conocimiento" (p.189).

\section{CONCLUSIONES}

El uso del software GeoGebra como medio dinámico en el aprendizaje de la geometría motiva a los estudiantes a interactuar y manipular las herramientas del programa lo que lleva a potenciar las competencias matemáticas $y$ fortalecer el trabajo en grupo entre los estudiantes.

Desde la Teoría de las Situaciones Didácticas y el modelo de Van Hiele se diseñaron situaciones didácticas que permitieron a los estudiantes movilizarse por diferentes momentos del desarrollo de la actividad, utilizar sus saberes previos aceptando o rechazando estrategias de solución, intercambiando los 
resultados encontrados con otros estudiantes y demostrando la validez de los mismos para formalizar el conocimiento matemático adquirido.

Se concluye, por tanto, que es importante implementar en el diseño de las secuencias didácticas, situaciones adidácticas, ya que estas permiten a los estudiantes la construcción autónoma del saber matemático, además permiten fortalecer el aprendizaje geométrico y el trabajo en grupo, aspectos que quedaron evidenciados en esta investigación como dificultades que se deben fortalecer en los estudiantes en las clases.

\section{REFERENCIAS BIBLIOGRÁFICAS}

Arias, F. (2012). El Proyecto de Investigación. Introducción a la metodología científica. Caracas - República Bolivariana de Venezuela: Editorial EPISTEME, C.A.

Brousseau, G. (2007). Iniciación al estudio de las situaciones didácticas. Buenos Aires: Libros del Zorzal.

Chavarría, J. (2006). Teoría de las Situaciones Didácticas. Cuadernos de investigación y formación en educación matemática.

Chevallard, Y., Bosch, M., \& Gascón, J. (2005). Estudiar matemáticas. El eslabón perdido entre la enseñanza y el aprendizaje. España: Editorial Horsori.

Córdoba, F. J. (2014). Las TIC en el aprendizaje de las matemáticas: ¿ Qué creen los estudiantes? Congreso Iberoaméricano de Ciencia, Tecnología, Innovación y Educación.

Ezguerro, M. (2014). Uso de GeoGebra en la enseñanza de Geometría analítica en $4^{\circ}$ en la ESO. Galdakao (Viscaya): Universidad Internacional de la Rioja.

Fandiño, M. (2009). Las fracciones: aspectos conceptuales y didácticos. Bogotá:
Editorial Magisterio.

Fouz, F. (2013). Modelo de Van Hiele para la didáctica de la Geometría.

Gómez, N., Sánchez, D., \& Sepúlveda, O. (2021). La comprensión del movimiento rectilíneo a través de la representaciones semióticas. Revista Boletín Redipe, p. 195-204.

Hernandez, R., Collado, C. F., \& Baptista, M. (2014). Metodología de la Investigación. México, D.F: McGRAW -HILL/ Interamericana Editores, S.A DE C.V.

Leung, F. (2006). "The impact of information and communication Tecnology on Our Understading of the Nature of mathematics". . For the Learning of mathematics, p. 29-35.

Ministerio de Educación Nacional (MEN). (2006). Visión 2019: Educación para una discusión. Bogotá, D.C: Ministerio de Educación.

Said, E. (2015). Hacia el fomento de las TIC en el sector educativo Colombian. Barranquilla Colombia: Editorial Universidad del Norte.

Vargas, G., \& Gamboa, R. (2013). El modelo de Van Hiele y la enseñanza de la Geometría. UNICIENCIA Vol.27, $N^{\circ} 1$, p. 74-94. 